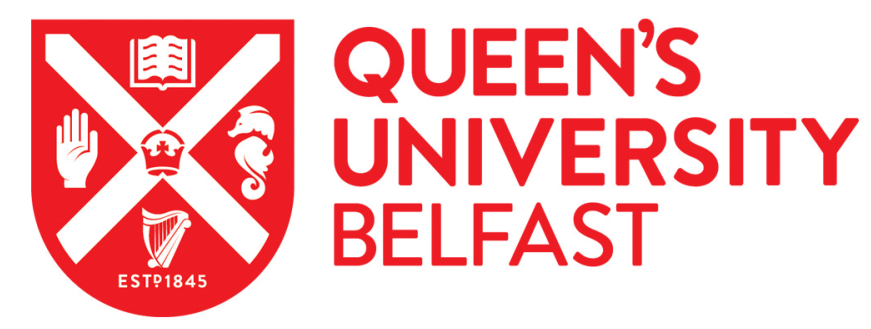

\title{
Microbial phylogenetic and functional responses within acidified wastewater communities exhibiting enhanced phosphate uptake
}

Weerasekara, A. W., Jenkins, S., Abbott, L. K., Waite, I., McGrath, J. W., Larma, I., Eroglu, E., O'Donnell, A., \& Whiteley, A. S. (2016). Microbial phylogenetic and functional responses within acidified wastewater communities exhibiting enhanced phosphate uptake. Bioresource Technology, 220, 55-61.

https://doi.org/10.1016/j.biortech.2016.08.037

Published in:

Bioresource Technology

Document Version:

Peer reviewed version

Queen's University Belfast - Research Portal:

Link to publication record in Queen's University Belfast Research Portal

Publisher rights

(c) 2016 Elsevier Ltd. This manuscript version is made available under the CC-BY-NC-ND 4.0 license http://creativecommons.org/licenses/by$\mathrm{nc}-\mathrm{nd} / 4.0 /$,which permits distribution and reproduction for non-commercial purposes, provided the author and source are cited.

\section{General rights}

Copyright for the publications made accessible via the Queen's University Belfast Research Portal is retained by the author(s) and / or other copyright owners and it is a condition of accessing these publications that users recognise and abide by the legal requirements associated with these rights.

Take down policy

The Research Portal is Queen's institutional repository that provides access to Queen's research output. Every effort has been made to ensure that content in the Research Portal does not infringe any person's rights, or applicable UK laws. If you discover content in the Research Portal that you believe breaches copyright or violates any law, please contact openaccess@qub.ac.uk. 


\section{Communities Exhibiting Enhanced Phosphate Uptake}

Anjani W. Weerasekara ${ }^{\mathrm{a}, \mathrm{b}}$, Sasha Jenkins ${ }^{\mathrm{a}, \mathrm{b}}$, Lynette K. Abbott ${ }^{\mathrm{a}, \mathrm{b}}$, Ian Waite ${ }^{\mathrm{a}, \mathrm{b}}$, John W. McGrath $^{\mathrm{d}}$, Irma Larma ${ }^{\mathrm{e}}$, Ela Eroglu ${ }^{\mathrm{f}}$, Anthony O'Donnell ${ }^{\mathrm{a}, \mathrm{b}}$ and Andrew S. Whiteley ${ }^{\mathrm{a}}$ Affiliations:

aschool of Earth and Environment, Faculty of Science, The University of Western Australia, 35 Stirling Highway, Crawley, 6009, Western Australia, Australia

${ }^{\mathrm{b}}$ Institute of Agriculture, The University of Western Australia, 35 Stirling Highway, Crawley 6009. Western Australia, Australia

${ }^{\mathrm{d} S c h o o l ~ o f ~ B i o l o g i c a l ~ S c i e n c e s ~ a n d ~ t h e ~ I n s t i t u t e ~ f o r ~ G l o b a l ~ F o o d ~ S e c u r i t y, ~ T h e ~ Q u e e n s ~}$ University of Belfast, Medical Biology Centre, 97 Lisburn Road, Belfast BT9 7BL, Northern Ireland

${ }^{\mathrm{e} C e n t r e ~ f o r ~ M i c r o s c o p y, ~ C h a r a c t e r i s a t i o n ~ a n d ~ A n a l y s i s ~(C M C A), ~ T h e ~ U n i v e r s i t y ~ o f ~}$ Western Australia, Crawley, 35 Stirling Highway, 6009, Western Australia, Australia ${ }^{\mathrm{f}}$ Department of Chemical Engineering, Curtin University, Perth, 6845, Western Australia, Australia

\section{*Author for Correspondence:}

\section{Prof. Andrew Whiteley (andy.whiteley@uwa.edu.au)}

School of Earth and Environment, Faculty of Science, The University of Western Australia, 35 Stirling Highway, Crawley, 6009, Western Australia, Australia 
ABSTRACT

Acid stimulated accumulation of insoluble phosphorus within microbial cells is highly beneficial to wastewater treatment but remains largely unexplored. Using single cell analyses and next generation sequencing, the response of active polyphosphate accumulating microbial communities under conditions of enhanced phosphorus uptake under both acidic and aerobic conditions was characterised. Phosphorus accumulation activities were highest under acidic conditions ( $\mathrm{pH} 5.5$ > 8.5), where a significant positive effect on bioaccumulation was observed at $\mathrm{pH} 5.5$ when compared to $\mathrm{pH} 8.5$. In contrast to the Betaproteobacteria and Actinobacteria dominated enhanced biological phosphorus removal process, the functionally active polyP accumulators at $\mathrm{pH} 5.5$ belonged to the Gammaproteobacteria, with key accumulators identified as members of the families Aeromonadaceae and Enterobacteriaceae. This study demonstrated a significant enrichment of key polyphosphate kinase and exopolyphosphatase genes within the community metagenome after acidification, concomitant with an increase in P accumulation kinetics. phosphorus removal / Polyphosphate kinase 


\section{Introduction}

Phosphorus is present at high loading in wastewater streams and is a key agent of environmental eutrophication. However, the impact of soluble phosphorus contained within release streams can be mitigated using technologies that induce enhanced phosphate uptake by the microbial community and its intracellular accumulation as insoluble polyphosphate (polyP) (McGrath and Quinn, 2003; Nielsen et al., 2010). Enhanced Biological Phosphorus Removal (EBPR) is an effective, low cost biotechnological process for P removal from wastewater which is achieved by modifying the conventional activated sludge system to include alternating anaerobic and aerobic phases (McGrath and Quinn, 2003; Yuan et al., 2012).

Although EBPR has been widely studied from the engineering and chemical perspective, an understanding of the microorganisms performing the $\mathrm{C}$ and $\mathrm{P}$ transformation involved in EBPR, together with the environmental factors affecting enhanced P (and polyP) accumulation efficiency, have yet to be fully resolved. Such knowledge is clearly fundamental to the design of effective P removal systems (Nielsen et al., 2010). Indeed, EBPR processes can show variation in the efficiency and effectiveness of P removal (Kawaharasaki et al., 1999; Oehmen et al., 2007). Functional EBPR systems depend on the interaction of complex microbial communities with the prevailing environment within a specific compartment of the treatment process.

Consequently, process optimisation is maintained through the control of the operational environment within the process compartments, but this can be difficult to do without a fundamental understanding of the types of microorganisms involved and their pathways under different environmental conditions, such as anaerobic and aerobic transitions. 
Therefore, the desire for simplified systems has promoted the analyses of simpler, alternative environmental conditions which can induce enhanced phosphorus uptake. PolyP biosynthesis is not just confined to those microorganisms exposed to the alternating anaerobic/aerobic environment of the EBPR treatment regime. Given the prevalence of polyP throughout the microbial world, it is clear that polyP accumulation can be triggered by many environmental factors (Powell et al., 2008; Zheng et al., 2014) and extensive microbial polyP accumulation has been measured in response to nitrogen, amino acid or P limitation as well as to osmotic or oxidative stress (Mullan et al., 2006; Rao et al., 2009; Temperton et al., 2011). Other evidence has shown that enhanced polyP accumulation can be induced in fully aerobic cultures, under acidic conditions, without the need for prior anaerobiosis (McGrath et al., 2001; Moriarty et al., 2006; Mullan et al., 2002). McGrath and Quinn (2000) reported a 10.5-fold increase in intracellular polyP accumulation in Candida humicola G-1, grown at $\mathrm{pH} 5.5$ versus $\mathrm{pH}$ 7.5. In operational mixed, aerobic wastewater communities of activated sludge, phosphate uptake increased between $50 \%$ and $143 \%$ when the $\mathrm{pH}$ was adjusted down to 5.5 as compared to normal operating levels around pH 7.5 (McGrath et al., 2001). In these acidic systems, 34\% of the activated sludge microflora proved to be capable of increased phosphate uptake following acidification. These findings showed that phosphate removal could be substantially enhanced by acidification under strictly aerobic conditions (McGrath et al., 2001; Moriarty et al., 2006; Mullan et al., 2002) and that the process was economically feasible (Mullan et al., 2006). Duguid et al. (1954) have previously reported that intracellular polyP production in Klebsiella aerogenes occurred only during growth at $\mathrm{pH} 4.0$ to 5.0 and not at neutral $\mathrm{pH}$ values. Similar observations have been made in soil fungi where Gerlitz (1996; 1997) demonstrated 
that maximal polyP accumulation in the ectomycorrhizal fungus Suillus bovines was 35\% greater at $\mathrm{pH} 5.5$ than at $\mathrm{pH}$ 7.5. However, information on how acidic conditions influence microbial community dynamics and the bioaccumulation of polyP during wastewater treatment acidic remains largely unexplored.

$$
\text { Using previous observations of acid stimulated } \mathrm{P} \text { removal and polyP accumulation, }
$$
we sought to understand the mechanism and taxa involved in engineered biological systems which could accumulate phosphorus under these conditions. It was hypothesised that there would be a significant microbial polyphosphate accumulation under acid conditions in engineered wastewater treatment communities and that this would be accompanied by an increase in functionally active polyP accumulators and $\mathrm{P}$ accumulation kinetics. To understand the microbial communities involved, a combined phylogenetic and metagenome analysis was used to investigate changes in the bacterial community structure (16S rRNA genes) and functional diversity (metagenomics) of waste treatment systems run under acidic and near-neutral conditions. These changes in structural and functional diversity were evaluated relative to increases in P uptake. To quantify the organisms responsible for poly P accumulation, flow cytometry and coincident single cell analyses was used to enumerate functional polyphosphate accumulators coupled with next generation sequencing to determine the community structure and diversity of the organisms involved. The approach used provides a 'cell to population' understanding of the polyP accumulation process that can then be used as the basis of an optimisation strategy for acid stimulated P removal from wastewater streams. 


\section{Methods}

121

122

123

124

125

126

127

128

\subsection{Sampling site description and sampling}

A covered anaerobic pond (CAP) fitted with an impermeable cover was constructed at Medina Research Station, Western Australia (GPS geocoder: Latitude -32.223000, Longitude 115.805801) to treat piggery effluent waste and capture bioenergy. The waste treatment process can be separated into 5 stages: collection pits in the pig shed; solid separation screens, holding tank, the CAP and finally a secondary aerobic pond.

Effluent from the pig pens was collected into the pits followed by release into a 100,000

L underground tank from where it was pumped over a static run-down screen (solid separator) that removed $10-15 \%$ of the total solids. The remaining wastewater was transferred to a holding tank prior to being pumped into the CAP digester (ca. 25m $\mathrm{x} 25 \mathrm{~m} \mathrm{x} 5 \mathrm{~m})$ on a weekly basis $(75,000 \mathrm{~L} / \mathrm{wk})$. The biogas produced from the CAP was removed through a perforated pipe system placed around the perimeter of the pond. Treated effluent was finally transferred to the secondary aerobic pond (ca. 50m x50m x5m) for evaporation (Fig. S1).

Samples for laboratory phosphorus removal experiments were collected from the aerobic pond by suction using a $12 \mathrm{~V}$ marine grade bilge pump connected to a PVC hosepipe. The hosepipe was placed into the aerobic pond and run for 5 mins to flush the sampling line, and samples from each point were collected into several autoclaved containers with corresponding samples mixed together to make a composite sample for laboratory incubation experiments. The chemical composition of the composite wastewater was assessed as follows: pH 8.5; EC $5.2 \mathrm{mS} / \mathrm{cm}$; chemical oxygen demand $483 \mathrm{mg} / \mathrm{L}$; total solids $0.1 \%$; volatile solids $25.8 \%$; total nitrogen $6.8 \%$; total C 45.9 \%, total P 13.6 mg/ L; orthophosphate 12.2 mg/L; Ca 21 mg/L; Mg 148 mg/L; K 681 
$\mathrm{mg} / \mathrm{L} ; \mathrm{Fe} 0.1 \mathrm{mg} / \mathrm{L}$ using standard methods for the analysis of water and wastewater

145 (Eaton et al., 2005).

\subsection{Lab-scale incubation experiment}

The initial $\mathrm{pH}$ and inorganic orthophosphate concentration (Pi) of the wastewater at the sampling was $\mathrm{pH} 8.5$ and $12.2 \pm 0.3 \mathrm{mg} / \mathrm{L}$ respectively. The Pi concentration was adjusted to $25 \mathrm{mg} / \mathrm{L}$ using $\mathrm{KH}_{2} \mathrm{PO}_{4}(25 \mathrm{mM})$ to simulate a moderately low $\mathrm{P}$ loaded wastewater system for lab-scale evaluation. The experimental design comprised of five different $\mathrm{pH}$ treatments $(5.5,6.0,6.5,7.0$ and 8.5 [control]) run in triplicate to determine the best $\mathrm{pH}$ level for polyP accumulation. The control represented the natural $\mathrm{pH}$ level of the wastewater at the time of sampling ( $\mathrm{pH}$ 8.5). For each treatment, effluents (300 $\mathrm{mL}$ ) were placed into autoclaved jars $(500 \mathrm{~mL})$ and kept under aerobic conditions (regular oxygen bubbling) at room temperature $\left(25^{\circ} \mathrm{C}\right)$ for $48 \mathrm{~h}$ under a natural 48h of incubation was determined using published methods (Eaton et al., 2005). Microcosm samples from pH 5.5 and 8.5 were chosen, after initial determination of optimum acidification conditions (Fig. S2), for downstream epi-fluorescence microscopy, flow cytometry and molecular analyses.

\subsection{Sample preparation for epi-fluorescence microscopy and flow cytometry}

At the end of the incubation experiment $(48 \mathrm{~h})$, aliquots $(1 \mathrm{~mL})$ from each microcosm were taken and centrifuged at $5000 \mathrm{x} \mathrm{g}$ for $5 \mathrm{~min}$. The cell pellet was washed with phosphate buffered saline (PBS) then resuspended in PBS, fixed with 4\% $(\mathrm{w} / \mathrm{v})$ paraformaldehyde fixative solution (PFA) and incubated overnight at $4^{\circ} \mathrm{C}$. For samples analysed by flow cytometry, cells were stained with 15 ug/mL DAPI (4',6-diamidino-2-phenylindole) for 20 mins, according to Kawaharasaki et al. (1999). 
For epi-fluorescence microscopy, the PFA fixed cells were washed with PBS and distilled water before mounting fifty microliters $(50 \mu \mathrm{L})$ of cell suspension in the middle of a microscope slide and air drying. Slides were subsequently stained with DAPI (15 ug/mL for 20 mins), rinsed with distilled water and airdried. Cells were visualised under x100 objective using a Zeiss Axioplan epifluorescence microscope under UV excitation

173 (DAPI filter block).

Flow cytometric analyses were performed using a BD Influx cell sorter (Becton

Dickinson, USA) at the Centre for Microscopy, Characterisation and Analyses at The University of Western Australia. DAPI was excited with a 355 nm (UV) laser with standard DAPI emission collected with a 460/50 nm band pass filter; the yellow shifted DAPI-PolyP bound emission was collected with a 585/29 bandpass filter.

Measurements for DAPI and polyP were acquired on a logarithmic scale and postacquisition analysis performed using Flow Jo software version 7.6.5. Single cells were gated on forward scatter area (FSC-A) vs forward scatter height (FSC-H) to exclude any doublets and DAPI-DNA and DAPI-polyP were gated to determine proportions of bacteria accumulating polyphosphate. Briefly, DAPI-bound polyP cell populations formed a distinct cluster in the polyP 570-600nm range whilst non-accumulators column purification, as described by the manufacturer. Extracted DNA was quantified and checked for purity at A260/280 nm (Nanodrop, Thermo Fisher Scientific, USA) 
polymerase chain reaction (PCR) from the DNA samples using Golay barcodes fused to Ion Torrent adapter modified core primers 341F and 518R (Whiteley et al., 2012), using amplification conditions described previously (Jenkins et al., 2010). All PCR products were checked for size and specificity by electrophoresis on 1.5\% w/v agarose, gel purified and adjusted to $10 \mathrm{ng} / \mu \mathrm{L}$ using molecular grade water and then pooled equally for subsequent sequencing. Sequencing was performed using an Ion Torrent Personal Genome Machine (PGM) (Life technologies, USA) using 200 base-pair chemistry as described previously by Whiteley et al. (2012). All the PGM quality filtered data were exported as FastQ files and split into *.fasta and *.qual files and analysed using the QIIME pipeline (Caporaso et al., 2010). Assigning the multiplexed reads to samples was performed using standard parameters (minimum quality score $=25$; minimum/maximum length = 130/220; no ambiguous base calls; removal of reverse primers; and no mismatches allowed in the forward and reverse primer sequences). Chimera checking was done using USEARCH61 (Edgar et al., 2011) and only nonchimeric sequences were assigned operational taxonomic units (OTUs) using the Greengenes (GG) reference database with clustering at 97\% identity using the UCLUST algorithm. Singletons were removed and taxonomy was assigned to the representative sequence of each OTU. Alpha rarefaction was performed using the phylogenetic diversity, Chao1, the Shannon Index and Observed Species metrics.

\subsection{Whole genome shotgun sequencing}

DNA was extracted from the laboratory microcosm experiments (pH 5.5 and $\mathrm{pH}$ 8.5) using the MoBio Powersoil DNA isolation kit (Geneworks, Australia), as described above. Genomic DNA sequencing of these samples was performed using whole genome shotgun sequencing where 150 ng of DNA was used to generate a whole genome 
216 shotgun library using a NEBnext Ultra library preparation kit (New England

217 Biosciences). Fragments of 320-330bp were selected from the final library by gel-

218 excision and sequenced for 520 flows on a Proton sequencer (Life Technologies),

219 yielding reads of 230-240bp modal length. Quality filtering and trimming were

220 performed 'on instrument' using TorrentSuite 4.0. The sequencing data for each of the

221 sampling points were rarefied to the same sequencing depth (300000 reads) and

222 uploaded to MG-RAST (Metagenome Rapid Annotation using Subsystem Technology

223 (MG-RAST) server (http://metagenomics.nmpdr.org/). Metagenomic data sets are

224 publicly available in the MG-RAST system under project identifiers 4553566.3 (pH 8.5;

225 control) and 4553567.3 (pH 5.5). Assignment of metabolic function and phylogenetic

226 identification were performed as described previously (Meyer et al., 2008).

227 2.6. Statistical analysis.

228 ANOVA was performed using the Statistical Analysis System (SAS) version 9.2

229 software package (SAS Institute, Inc. Cary, NC, USA). Means were separated using

230 least significant difference (LSD) at 5\% significance level.

\section{Results and Discussion}

\subsection{PolyP accumulation in a laboratory incubation experiment}

Soluble orthophosphate concentrations within aerobic microcosms indicated the absolute removal of $\mathrm{P}$ was $45 \%$ higher at $\mathrm{pH} 5.5$ than at $\mathrm{pH} 8.5$ after 48 hours (Fig. S2).

236 Subsequently, the observed increases in polyP accumulation were matched by an increase in the number of microorganisms able to accumulate polyP, as determined by direct epi-fluorescence microscopy and flow cytometry. Epifluorescence microscopy (Fig. S3) showed that qualitatively, the number of DAPI-polyP stained cells increased at 
pH 5.5 (Fig. S3b) when compared to pH 8.5 (Fig. S3a). Flow cytometric analyses for quantification of polyP accumulators (Fig.1a and 1b) indicated increased P accumulation was mediated by a doubling of the cells actively accumulating polyP at pH 5.5 when compared to pH 8.5 (70\% versus 36\% respectively). This was consistent with previous findings in other treatment systems, where growth of polyphosphate accumulators was enhanced and the aerobic uptake of phosphate reached a maximum at pH 5.5 (McGrath et al., 2001; Mullan et al., 2002; Moriarty et al., 2006).

3.2. Phylogenetic community structure of polyphosphate accumulating microorganisms under enhanced accumulation conditions

DNA extracted from incubated microcosms was used to assess the phylogenetic affiliations and relative abundances of the bacteria in microcosms incubated at $\mathrm{pH} 5.5$ and $\mathrm{pH} 8.5$ by next generation sequencing. After normalising all samples to 5000 sequence reads, alpha diversity metrics indicated that bacterial diversity was higher in samples incubated at pH 8.5 (246 OTUs) when compared to pH 5.5 (173 OTUs), indicating that acidification selected a sub-set of the diversity present within the control community structure at pH 5.5 when compared to pH 8.5 (Fig. 2). The bacterial community composition under 'normal' pH 8.5 operating conditions was dominated by Actinobacteria (50.7\%), followed by Betaproteobacteria (19.9\%), Erysipelotrichi (8.2 \%), TM7 (5.1\%) and Gammaproteobacteria (2.9\%). In comparison, members of the 260 Gammaproteobacteria accounted for $90 \%$ of the sequences recovered at $\mathrm{pH}$ 5.5. This indicated a 30 fold increase in Gammaproteobacteria taxa, represented by Aeromonadaceae (72\% of sequences classified), Enterobacteriaceae (16\%), 
264 Acinetobacter (2\%). Although previous work has shown that members of the

265 Gammaproteobacteria are effective polyP accumulating bacteria and have been

266 detected in other wastewater treatment systems (Nielsen et al., 2010) the most

267 commonly reported polyP accumulating organisms in both full scale EBPR systems and

268 laboratory EBPR bioreactors are from lineages of the Betaproteobacteria and

269 Actinobacteria, in particular Candidatus accumulibacter and Tetrasphaera respectively

270 (Maszenan et al., 2000; McMahon and Reid 2013). For cultured isolates, polyP

271 synthesis has been studied extensively in a wide range of microorganisms including

272 Escherichia coli, Saccharomyces cerevisiae and Helicobacter pylori (Rao et al., 2009)

273 and is well known within the Actinobacteria, Bacteroidetes, and Alpha-Beta- and

274 Gammaproteobacteria (McMahon and Read, 2013). This emerging evidence suggests

275 that microbial polyP accumulation is likely ubiquitous, and enrichment for r-selected

276 polyP accumulators such as the fast growing Gammaproteobacteria can occur under

277 relatively simple selection pressure, such as acidification. This contrasts with the much

278 more complex engineering requirements needed to impose anaerobic-aerobic cycling in

279 EBPR systems and indicates that acidification clearly represents a much more

280 simplified method to stimulate phosphate uptake.

281 3.3. Metabolic reconstruction of enhanced polyP accumulation by shotgun

282 metagenomics

283 Shotgun metagenomics was used to assess the presumptive functional genes involved

284 in aerobic acid stimulated polyP accumulation. The genetic potential for phosphorus

285 metabolism by the community, in terms of the richness and changes in the abundance of

286 genes involved in polyP metabolism, was compared by assigning functional annotations

287 to metagenomic sequences with subsequent sequence assignment to subsystems. The 
genetic potential for phosphorus metabolism was demonstrated by comparing changes (elevation) in the polyphosphate kinase1 (ppk1: EC 2.7.4.1) and exopolyphosphatase (ppx; EC 3.6.1.11) genes at pH 5.5 and 8.5; both genes are considered essential in polyP synthesis and hydrolysis, respectively (Rao et al., 2009; Fig. 3). Under acidified conditions, ppk1 was the most abundant gene sequence when compared to all other genes involved in phosphorus metabolism. Reid et al. (2008) also found that ppk1 dominated when Campylobacter jejuni was subject to acid shock. Furthermore, prior to polyP synthesis, phosphate is taken up and transported across the cytoplasmic membrane, a process governed by a number of enzymes and regulons (Lamarche et al., 2008). For these transport systems, metagenomic analyses indicated that the low affinity

Pit system (phosphate inorganic transport) and high affinity Pst (phosphate specific transport) systems (PstA, PstB, and PstC), were higher at $\mathrm{pH} 5.5$ than at $\mathrm{pH} 8.5$ (Fig. 3).

These data indicate that the selective enrichment for organisms that contained both phosphate uptake and polyP synthesis systems are more pronounced under acid conversion.

For polyP conversion, the bacterial ppk1 gene encoding for the enzyme polyphosphate kinase is responsible for polyP synthesis in many bacteria (Mielczarek et al., 2013). There was an increase in the relative gene abundance of ppk genes at pH 5.5 that were characteristic of homologs from Aeromonas hydrophila, Aeromonas salmonicida, Enterobacter sp., Pseudomonas aeruginosa, Klebsiella variicola, 
related to Bordetella avium, Burkholderia mallei, Kribbella flavida, Kineococcus radiotolerans, Cellulomonas flavigena and Chromobacterium were most abundant (Table 1). Thus, based upon this evidence coupled with the 16S rRNA studies, it would appear that acidification is selecting for the Gammaproteobacteria and their pathways as key agents in the enhanced $\mathrm{P}$ uptake and polyP accumulation. This is in contrast to EBPR processes that appear to be driven by representatives of the Betaproteobacteria and Actinobacteria (McMahon and Read, 2013). These community differences may explain the results of Filipe et al. (2001) who report a 53\% decrease in phosphate uptake during EBPR at pH 6.5. This contrast leads to the hypothesis that a drop in P uptake in normal EBPR systems at low pH may be due to the absence of Gammaproteobacteria capable of low $\mathrm{pH}$ accumulation, whereas these taxa are abundant in our study and correspond with species known to possess sequences homologs to ppk (Rao et al., 2009).

The exact reason for polyP accumulation under acidic conditions and its subsequent physiological role is still unclear. One hypothesis which deserves future attention is that enhanced polyP accumulation may help regulate intracellular phosphate and $\mathrm{pH}$ levels (Harold, 1966). For example, acid pH optimums for cellular P transport have been shown in Saccharomyces cerevisiae (pH 5.5: Borst-Pauwels and Peters, 1977), Yarrowia lipolytica (pH 4.5: Zvyagilskaya et al., 2000) and Burkholderia cepacia (Moriarty et al., 2006) which cause both an increase in cellular P and a decrease in cellular $\mathrm{pH}$. Conversion of excess phosphorus entering into the cell under optimum transport levels may allow a mechanism of homeostasis whereby any excess $\mathrm{P}$ can be effectively stored and the resulting polyP further acts as a buffer for cellular $\mathrm{pH}$ by acting as an intracellular cation trap, sequestering $\mathrm{H}^{+}$ions. Circumstantial evidence that 
this strategy may be in operation and deserves further attention is that polyP has

337 previously been linked to cellular $\mathrm{pH}$ homeostasis in both the unicellular alga

Dunaliella salina (Bental et al., 1991) and in Saccharomyces cerevisiae (Castro et al., 1995) whilst Lactococcus ppk- mutants show diminished growth at acid pH (Alcántra et al., 2014). However, irrespective of the physiological reasons for PolyP accumulation in response to acid $\mathrm{pH}$, it is demonstrated that this phenomenon may have significant implications for overall ecological P-cycling and the applied aspects of developing alternative technologies for P removal from waste streams. natural ecosystems, such as those microbial communities responsible for $\mathrm{P}$ cycling treatment systems. For the first time in an applied context, these data generated the fundamental understanding of how acid stimulation modulates P transformation. This as well as the potential for bioengineering the wastewater community for optimal activity and P recovery. 


\section{Conclusions}

This study demonstrated the microorganisms capable of mediating enhanced phosphorus removal from wastewater systems under acid stimulation and the genetic basis for this enhanced P accumulation. Both the phylogenetic and metagenomic analyses indicate increases in polyP accumulation are the result of substantial population increases in Gammaproteobacteria taxa, in tandem with an enhanced community genetic capacity for both cellular P transport and polyP production. These community shifts result in a substantial quantitative population increase in $\mathrm{P}$ removal capacitywhich can be visualised within the accumulator cells as insoluble P deposition as acid stimulation modifies the nascent community structure and function.

Acknowledgements

This work was funded by grants from Australia Pork Ltd and the Australian Grains Research and Development Corporation (GRDC). The authors would also like to acknowledge the facilities, and the scientific and technical assistance of the Australian Microscopy and Microanalysis Research Facility at the Centre for Microscopy, Characterisation \& Analysis (CMCA), The University of Western Australia (UWA), which is funded by the University and by State and Commonwealth Governments. The authors would like to acknowledge Dr. Kathy Heel for her assistance in Flow cytometry, Dr Richard Allcock for sequence generation and Dr.Falko Mathes for his input during the sequencing analysis. ASW is supported by the 2012 West Australian Fellowship Program award, funded jointly by the Department of Premier and Cabinet, WA and The University of Western Australia. 


\section{References}

1. Alcántara, C., Blasco, A., Zúñiga, M., Monedero, V., 2014. Accumulation of Polyphosphate in Lactobacillus spp. and its involvement in stress resistance. Appl. Environ. Microbiol. 80, 1650-1659.

2. Bental, M., Pick, U., Avron, M., Degani, H., 1991. Polyphosphate metabolism in the alga Dunaliella salina studied by P31-NMR. Biochim. Biophys. Acta. 1092, 21-28.

3. Borst-Pauwels, G.W.F.H., Peters, P.H.J., 1977. Effect of the medium pH and the cell $\mathrm{pH}$ upon the kinetical parameters of phosphate uptake by yeast. Biochim. Biophys. Acta. 466, 488-495.

4. Caporaso, J.G., Kuczynski, J., Stombaugh, J., Bittinger, K., Bushman, F.D., Costello, E.K., Fierer, N., Pena, A.G., Goodrich, J.K., Gordon, J.I., 2010. QIIME allows analysis of high-throughput community sequencing data. Nat. Methods. 7, 335-336.

5. Castro, C.D., Meehan, A.J., Koretsky, A. P., Domach, M. M., 1995. In situ ${ }^{31}$ P nuclear magnetic resonance for observation of polyphosphate and catabolite responses of chemostat-cultivated Saccharomyces cerevisiae after alkalinization. Appl. Environ. Microbiol. 61, 4448-4453.

6. Duguid, J. P., Smith, I.W., Wilkinson, J.F., 1954. Volutin production in Bacterium aerogenes due to development of an acid reaction. J. Path. Bact. 67.289-300.

7. Eaton, A.D., Clesceri, L.S., Greenberg, A.E., 2005. Standard methods for the examination of water and wastewater American Public Health Association. Washington, DC, 20001-23710. 
8. Edgar, R.C., Haas, B.J., Clemente, J.C., Quince, C., Knight, R., 2011. UCHIME improves sensitivity and speed of chimera detection. Bioinformatics 27, 21942200.

9. Filipe, C.D.M., Daigger, G.T., Grady, C.P., 2001. Effects of pH on the rates of aerobic metabolism of phosphate-accumulating and glycogen-accumulating organisms. Water Environ. Res. 73, 213-222.

10. Gerlitz, T.G.M., 1996. Effects of aluminium on polyphosphate mobilization of the ectomycorrhizal fungus Suillus bovinus. Plant Soil 178, 133-140.

11. Gerlitz, T.G.M., Gerlitz, A., 1997. Phosphate uptake and polyphosphate metabolism of mycorrhizal and nonmycorrhizal roots of pine and of Suillus bovinus at varying external pH measured by in vivo P-31-NMR. Mycorrhiza 7, 101-106.

12. Harold, F.M., 1966. Inorganic polyphosphate in biology: structure, metabolism, and function. Bacteriol. Rev. 30, 772-794.

13. Jenkins, S.N., Rushton, S.P., Lanyon, C.V., Whiteley, A.S., Waite, I.S., Brookes, P.C., Kemmitt, S., Evershed, R.P., O’Donnell, A.G., 2010. Taxonspecific responses of soil bacteria to the addition of low level C inputs. Soil Biol. Biochem. 42, 1624-1631.

14. Kawaharasaki, M., Tanaka, H., Kanagawa, T., Nakamura, K., 1999. In situ identification of polyphosphate-accumulating bacteria in activated sludge by dual staining with rRNA-targeted oligonucleotide probes and 4', 6-diamidino-2phenylindol (DAPI) at a polyphosphate-probing concentration. Water Res. 33, 257-265. 
15. Lamarche, M.G., Wanner, B.L., Crépin, S., Harel, J., 2008. The phosphate regulon and bacterial virulence: a regulatory network connecting phosphate homeostasis and pathogenesis. FEMS Microbiol. Rev. 32, 461-473.

16. Maszenan, A.M., Seviour, R.J., Patel, B.K.C., Schumann, P., Burghardt, J., Tokiwa, Y., Stratton, H.M., 2000. Three isolates of novel polyphosphateaccumulating gram-positive cocci, obtained from activated sludge, belong to a new genus, Tetrasphaera gen. nov., and description of two new species, Tetrasphaera japonica sp. nov. and Tetrasphaera australiensis sp. nov. Int. J. Syst. Evol. Microbiol. 50, 593-603.

17. McGrath, J.W., Cleary, S., Mullan, A., Quinn, J.P., 2001. Acid-stimulated phosphate uptake by activated sludge microorganisms under aerobic laboratory conditions. Water Res. 35, 4317-4322.

18. McGrath, J.W., Quinn, J.P., 2000. Intracellular accumulation of polyphosphate by the yeast Candida humicola G-1 in response to acid pH. Appl. Environ. Microbiol. 66, 4068-4073.

19. McGrath, J.W., Quinn, J.P., 2003. Microbial phosphate removal and polyphosphate production from wastewaters. Adv. Appl. Microbiol. 52, 75-100.

20. McMahon, K.D., Read, E.K., 2013. Microbial contributions to phosphorus cycling in eutrophic lakes and wastewater. Annu. Rev. Microbiol. 67, 199-219.

21. Meyer, F., Paarmann, D., D'Souza, M., Olson, R., Glass, E.M., Kubal, M., Paczian, T., Rodriguez, A., Stevens, R., Wilke, A., 2008. The metagenomics RAST server-a public resource for the automatic phylogenetic and functional analysis of metagenomes. BMC Bioinformatics 9, 386. 
22. Mielczarek, A.T., Nguyen, H.T.T., Nielsen, J.L., Nielsen, P.H., 2013. Population dynamics of bacteria involved in enhanced biological phosphorus removal in Danish wastewater treatment plants. Water Res. 47, 1529-1544.

23. Moriarty, T., Mullan, A., McGrath, J., Quinn, J., Elborn, J., Tunney, M., 2006. Effect of reduced $\mathrm{pH}$ on inorganic polyphosphate accumulation by Burkholderia cepacia complex isolates. Lett. Appl. Microbiol. 42, 617-623.

24. Mullan, A., McGrath, J.W., Adamson, T., Irwin, S., Quinn, J.P., 2006. Pilotscale evaluation of the application of low $\mathrm{pH}$-inducible polyphosphate accumulation to the biological removal of phosphate from wastewaters. Environ. Sci. Technol. 40, 296-301.

25. Mullan, A., Quinn, J. P, McGrath, J.W., 2002. Enhanced phosphate uptake and polyphosphate accumulation in Burkholderia cepacia grown under low-pH conditions. Microbial ecol. 44, 69-77.

26. Nielsen, P.H., Mielczarek, A.T., Kragelund, C., Nielsen, J.L., Saunders, A.M., Kong, Y., Hansen, A.A., Vollertsen, J., 2010. A conceptual ecosystem model of microbial communities in enhanced biological phosphorus removal plants. Water Res. 44, 5070-5088.

27. Oehmen, A., Lemos, P.C., Carvalho, G., Yuan, Z., Keller, J., Blackall, L.L., Reis, M.A., 2007. Advances in enhanced biological phosphorus removal: from micro to macro scale. Water Res. 41, 2271-2300.

28. Powell, N., Shilton, A.N., Pratt, S. and Chisti, Y., 2008. Factors influencing luxury uptake of phosphorus by microalgae in waste stabilization ponds. Environ. Sci. Technol. 42, 5958-5962.

29. Rao, N.N., Gomez-Garcia, M.R., Kornberg, A., 2009. Inorganic polyphosphate: 
essential for growth and survival. Annu. Rev. Biochem. 78, 605-47.

479

480

481

482

483

484

485

486

487

488

489

490

491

492

493

494

495

496

497

498

499

500
30. Reid, A,N., Pandey, R., Palyada, K., Whitworth, L., Doukhanine, E., Stintzi, A., 2008. Identification of Campylobacter jejuni genes contributing to acid adaptation by transcriptional profiling and genome-wide mutagenesis. Appl. Environ. Microbiol. 74, 1598-1612.

31. Temperton, B., Gilbert, J.A., Quinn, J.P., McGrath, J.W., 2011. Novel analysis of oceanic surface water metagenomes suggests importance of polyphosphate metabolism in oligotrophic environments. PLoS One 6, e16499.

32. Whiteley, A.S., Jenkins, S., Waite, I., Kresoje, N., Payne, H., Mullan, B., Allcock, R., O'Donnell, A., 2012. Microbial 16S rRNA Ion Tag and community metagenome sequencing using the Ion Torrent (PGM) Platform. J. Microbiol. Methods. 91, 80-88.

33. Yuan, Z.G., Pratt, S., Batstone, D.J., 2012. Phosphorus recovery from wastewater through microbial processes. Curr. Opin. Biotechnol. 23, 878-883.

34. Zheng, X., Sun, P., Han, J., Song, Y., Hu, Z., Fan, H., Lv, S., 2014. Inhibitory factors affecting the process of enhanced biological phosphorus removal (EBPR) - A mini-review. Process Biochem. 49, 2207-2213.

35. Zvyagilskaya, R., Allard, P., Persson, B.L., 2000. Two systems for phosphate uptake in Yarrowia lipolytica cells grown at acidic conditions. IUBMB Life 49, 143-147. 
502 Fig. 1. Flow cytometric community analysis of polyP positive microbial populations

503 within wastewater communities at (a) $\mathrm{pH} 8.5$ and (b) $\mathrm{pH} 5.5$.

504 Fig. 2. Identities and relative abundance (\%) of the bacteria in laboratory microcosms 505 incubated at $\mathrm{pH} 8.5$ (natural $\mathrm{pH}$ of wastewater) and at $\mathrm{pH} 5.5$ (acidified wastewater). 506 Inset chart shows the composition of the Gammaproteobacteria at $\mathrm{pH}$ 5.5.

507 Fig. 3. Abundance of genes involved in the uptake and transport of inorganic phosphate 508 across the cytoplasmic membrane (low affinity Pit system and high affinity Pst), polyP synthesis (polyphosphate kinase), and hydrolysis (exopolyphosphatase) in laboratory microcosms at $\mathrm{pH} 8.5$ and $\mathrm{pH}$ 5.5. Polyphosphate kinase (ppk), phosphate transport system permease protein (pstC), a probable low-affinity inorganic phosphate transporter

512 (LAT), a phosphate transport ATP-binding protein (pstB), alkaline phosphatase ( $A L P L)$,

513 phosphate transport system permease protein ( $p s t A)$, exopolyphosphatase ( $p p x)$, phosphate regulon sensor protein (phoR), and phosphate regulon transcriptional regulatory protein $(p h o B)$.

517 Table 1. Functional affiliations of polyphosphate accumulating microorganisms and 518 their percentage identities to cultured strains based on the presence of the ppk genes in microcosms incubated at pH 5.5 and pH 8.5. 
523 Fig. S1. (a) Location of the study site. (b) The piggery waste treatment process at

524 Medina Research Station, Department of Agriculture and Food, Western Australia

525 (DAFWA) for treating piggery effluent waste and capture bio-energy.

526 Fig. S2. Overall phosphate removal efficiencies from laboratory microcosms incubated

527 at different $\mathrm{pH}$ treatments (pH 5.5, 6.0, 6.5, 7.0, 8.5). Error bars indicate the standard

528 deviation where points with different letters (A, B, C, D, and E) are significantly

529 different from each other $(\mathrm{P}<0.05)$.

530 Fig. S3. Epifluorescence micrographs of DAPI stained cells from laboratory

531 microcosms incubated at (a) the natural $\mathrm{pH}$ of wastewater at the time of sampling (pH

532 8.5) and (b) pH 5.5.

533

534

535

536

537

538

539 